\title{
Evaluación de prácticas de diseño y análisis de datos en psicología mediante centro de calificaciones en Enseñanza Virtual.
}

\author{
Evaluation of practical classes \\ of design and data analysis in \\ psychology from an assessment \\ center in a virtual environment.
}

Salvador Chacón-Moscoso

ORCID: https://orcid.org/0000-0002-6401-7384

Universidad de Sevilla.

Departamento de Psicología Experimental.

schacon@us.es

DOI: http://dx.doi.org/10.12795/9788447231003.089

Pp.: 1928-1945 


\section{Contexto de intervención}

En el contexto de la nueva enseñanza virtual implementada debido a la crisis COVID-19, el presente Ciclo de Mejora (en adelante CIMA) plantea como innovación la evaluación de las prácticas de Diseño y Análisis de Datos en Psicología II mediante el centro de calificaciones de la plataforma 'BlackBoard Collaborate Ultra' en enseñanza virtual de la Universidad de Sevilla.

Diseño y Análisis de Datos en Psicología II es una asignatura obligatoria de 20 del Grado en Psicología. El objetivo general de esta asignatura es aportar al alumnado contenidos conceptuales, procedimentales y actitudinales para el diseño, ejecución e interpretación de la estadística en la resolución de hipótesis empíricas.

En este caso el CIMA se aplica concretamente en las clases prácticas del grupo 1 de inglés, caracterizado porque la actividad docente se realiza plenamente en inglés en esta asignatura. Es un grupo reducido de unas 45 personas, y a su vez, dada la situación de alerta sanitaria, en las clases prácticas cómo máximo se permite que asistan entre 15 y 17 personas. Vamos a utilizar este contexto en positivo, como una circunstancia que posibilita un seguimiento continuado más efectivo que si el grupo de clase fuese mayor.

\section{Clases habituales}

En cursos anteriores, estas clases prácticas se ha desarrollado de la siguiente forma. Primero se hacía una breve descripción por parte del profesorado de los aspectos conceptuales y procedimentales de la clase para seguidamente pasar el alumnado a resolver ejercicios prácticos de forma individual o en grupo desarrollando su resolución bien manualmente o mediante el uso de software de análisis de datos.

Ciclos de Mejora en el Aula (2020). Experiencias de Innovación Docente de la US Esta obra se distribuye con la licencia Creative Commons 
Durante y después de cada resolución de cada ejercicio práctico, se pedía feedback al alumnado para confirmar que iba siguiendo la explicación; por ejemplo, razones de elegir el estadístico a aplicar, o forma de codificar las variables según diseño del estudio.

En este CIMA hemos intentado sacar partido a la nueva situación provocada por el COVID-19, implementando una dinámica de evaluación y seguimiento on-line de la resolución de ejercicios prácticos utilizando el centro de calificaciones de BlackBoard Collaborate Ultra en el presente curso 2020-21.

\section{Conexión del CIMA actual con el anterior}

En el curso anterior, mi intervención estuvo centrada en fomentar la participación en el grupo de clases teóricas de Psicometría (Chacón, 2019) y en el previo en el uso en clases prácticas de ejercicios de formato cerrado en Diseño y Análisis de Datos en Psicología I (Chacón, 2018).

Este curso cambio de asignatura (la intervención el curso pasado se ejecutó en Psicometría y este año en Diseño y Análisis de Datos II). Psicometría tiene puntos en común con ésta, dado que ambas son de la misma área y se trabaja comprensión de conceptos estadísticos, cálculos y aplicaciones. Por tanto las conclusiones obtenidas en los dos cursos anteriores han servido para el CIMA actual. Se trata de fomentar la participación y de implementar una nueva herramienta de evaluación en las prácticas de Diseño y Análisis de Datos II. Es de resaltar que el $80 \%$ del alumnado formó parte del estudio previo, ya que el alumnado que cursa las asignaturas en inglés se mantiene en los grupos de inglés hasta finalizar el grado.

Ciclos de Mejora en el Aula (2020). Experiencias de Innovación Docente de la US Esta obra se distribuye con la licencia Creative Commons 
En el nuevo diseño mantengo los principios didácticos del CIMA anterior de promover la participación activa (García-Pérez y Porlán, 2017) y la motivación del alumnado. En el caso de las clases prácticas es mucho más factible de conseguir que en la clases teóricas al implicar como diseño de partida el trabajo del alumnado.

\section{Diseño previo del CIMA}

\section{Mapa de contenidos y problemas claves}

Siguiendo las directrices presentadas en García-Díaz, Porlán y Navarro (2017), se ha realizado la Figura 1 que representa los contenidos fundamentales que el alumnado debe adquirir y preguntas clave relacionadas.

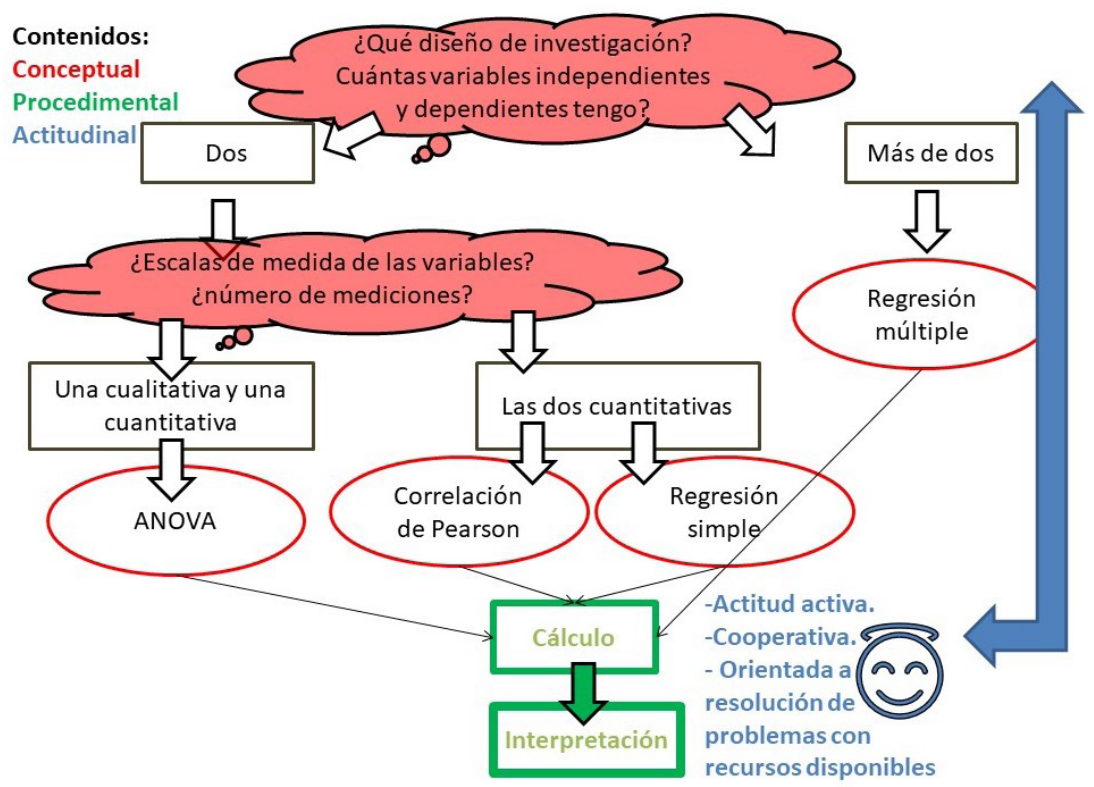

Figura 1. Mapa de contenidos y preguntas-clave Ciclos de Mejora en el Aula (2020). Experiencias de Innovación Docente de la US Reconocimiento-NoComercial-SinObraDerivada 4.0 Internacional (CC BY-NC-ND 4.0.) 
Los contenidos conceptuales, procedimentales y actitudinales están muy relacionados. Los colores en la Figura 1 indican de qué tipo de contenido se trata.

En el caso de las clases prácticas los contenidos y desarrollo de las mismas están muy relacionados con las clases teóricas. No obstante, en este tipo de clases ha dado, aún más, especial importancia al fomento del aprendizaje autónomo y de una actitud favorable hacia los contenidos conceptuales y procedimentales, mediante una actitud activa, colaborativa y orientada a la resolución de problemas mediante todos los recursos disponibles.

\section{Modelos ideal y posible, y secuencia de actividades}

En el modelo metodológico ideal, la función del profesorado en las clases prácticas es sólo de orientador y organizador, y es el alumnado el que presenta un rol protagonista y activo en su propio aprendizaje autónomo y tiene una actitud favorable y un alto grado de motivación por la asignatura (Polanco-Hernández, 2005). Al final de curso será capaz de traducir los distintos problemas de intervención a terminología estadística y con la posibilidad de transferencia a la actuación profesional.

En el modelo metodológico posible se harán actuaciones para facilitar la participación de todos y todas y así obtener a diario información sobre cómo se va desarrollando el aprendizaje del alumnado a modo de seguimiento. Aprovechando el uso de la plataforma Blackboard Collaborate Ultra, se le ha pedido al alumnado que entregue en el centro de calificaciones, ya sea en grupo o individualmente los resultados y resoluciones de los ejercicios prácticos desarrollados en clase. De esta forma el alumnado ha recibido una calificación automática y tendrá un feedback directo e inmediato de lo desarrollado en clase. Esto tendrá una

Ciclos de Mejora en el Aula (2020). Experiencias de Innovación Docente de la US Esta obra se distribuye con la licencia Creative Commons 
repercusión directa sobre la calificación final, para el caso del alumnado que opta por la evaluación continua. Todo esto ya está recogido en el proyecto docente de la asignatura, aprobado en consejo de departamento. La Figura 2 representa las discrepancias entre ambos modelos, representándose de nuevo en rojo los contenidos conceptuales, en verde los procedimentales y en azul los actitudinales.

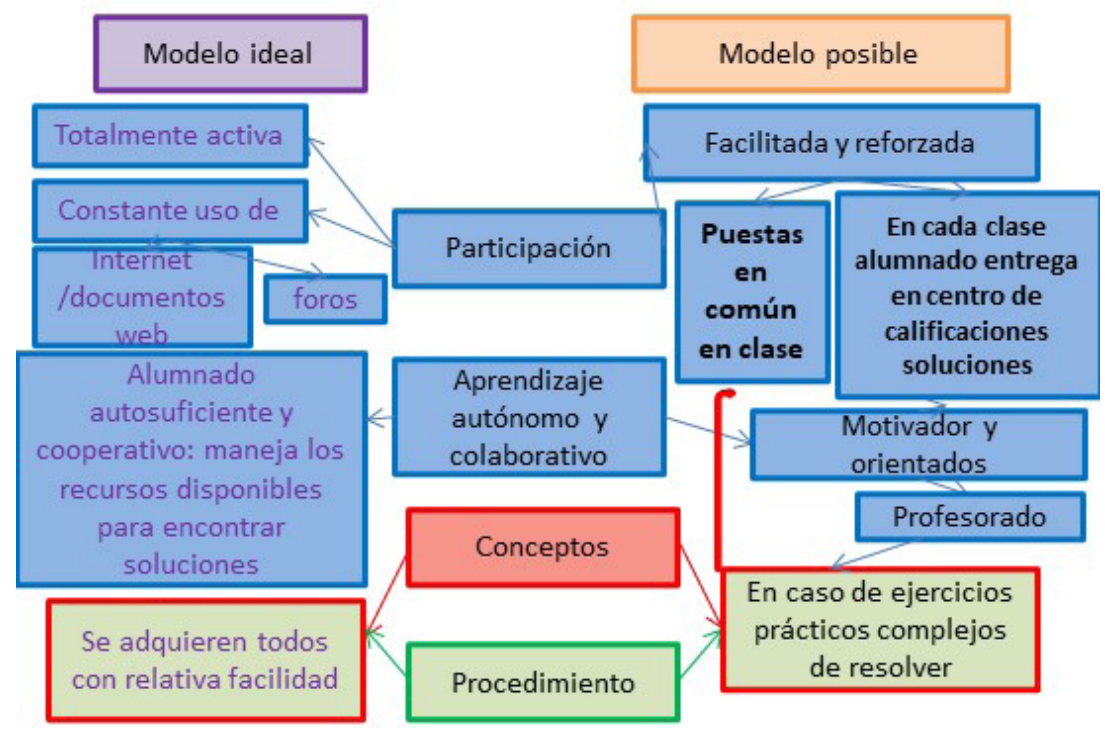

Figura 2. Modelos ideal y posible

La secuencia de actividades se ha desarrollado a lo largo de las clases prácticas. Se le ha pedido al alumnado que entregue en el centro de calificaciones, individualmente los resultados y resoluciones de los ejercicios prácticos. De igual forma se ha fomentado, que bien individual o en forma grupal el alumnado exponga en clase cómo ha orientado la actividad y cómo la ha resuelto. En la siguiente dirección web se presenta una relación posibles actividades a desarrollar en las clases prácticas: http://innoevalua.us.es/wakka. php?wakka=DesignAndDataAnalysisInPsychology\&v=16rv

Ciclos de Mejora en el Aula (2020). Experiencias de Innovación Docente de la US Esta obra se distribuye con la licencia Creative Commons Reconocimiento-NoComercial-SinObraDerivada Internacional (CC BY-NC-ND 4.0.) 
A continuación, se presentan dos casos prácticos planteados a modo de ejemplo de posibles actividades a desarrollar en clases prácticas:

Ejemplo de actividad 1:

Please, answer questions $1-4$ based on the following data:

We studied the relationship between level of extrovertion (high, medium, low) on the afection concern in a sample of 80 participants. The table below presents the coding used for each group and their means in afection concern:

\begin{tabular}{|c|c|c|c|}
\hline & $x_{1}$ & $x_{2}$ & $\bar{Y}$ \\
\hline High & 1 & 0 & 80 \\
\hline Medium & 0 & 0 & 100 \\
\hline Low & 0 & 1 & 70 \\
\hline
\end{tabular}

1. In the equation regression, $b_{0}$ is:
a) 100
b) 80
c) 90

2. In the equation regression, $b_{1}$ is:
a) -20
b) 10
c) -30

3) In the equation regression, $b_{2}$ is:
a) -20
b) 10
c) -30

4. The error for a low level of extrovertion of a person that obtained a score of 100 in afection concern is:

Ciclos de Mejora en el Aula (2020). Experiencias de Innovación Docente de la US Esta obra se distribuye con la licencia Creative Commons 

a) -10
b) 30
c) -30

Ejemplo de actividad 2:

Please, answer questions 5 - 9 based on the following data:

We studied if the anxiety level varied across the time applying different psychological treatments to participants. We obtained the following results:

Tests of Within-Subjects Effects

Measure: Anxiety

\begin{tabular}{|c|c|c|c|c|c|c|}
\hline & Source & SS & $d f$ & MS & $\mathrm{F}$ & Sig. \\
\hline $\begin{array}{l}\text { Moment } \\
\text { Measurement }\end{array}$ & $\begin{array}{l}\text { Sphericity Assumed } \\
\text { Greenhouse-Geisser } \\
\text { Huynh-Feldt } \\
\text { Lower-bound }\end{array}$ & $\begin{array}{l}1216.800 \\
1216.800 \\
1216.800 \\
1216.800 \\
\end{array}$ & $\begin{array}{c}1 \\
1.000 \\
1.000 \\
1.000 \\
\end{array}$ & \begin{tabular}{|l|}
1216.800 \\
1216.800 \\
1216.800 \\
1216.800 \\
\end{tabular} & $\begin{array}{l}173.829 \\
173.829 \\
173.829 \\
173.829 \\
\end{array}$ & $\begin{array}{l}.000 \\
.000 \\
.000 \\
.000 \\
\end{array}$ \\
\hline $\begin{array}{l}\text { Moment } \\
\text { Measurement } \\
\text { * Treatment }\end{array}$ & $\begin{array}{l}\text { Sphericity Assumed } \\
\text { Greenhouse-Geisser } \\
\text { Huynh-Feldt } \\
\text { Lower-bound }\end{array}$ & $\begin{array}{l}51.200 \\
51.200 \\
51.200 \\
51.200 \\
\end{array}$ & $\begin{array}{c}1 \\
1.000 \\
1.000 \\
1.000 \\
\end{array}$ & \begin{tabular}{|l|}
51.200 \\
51.200 \\
51.200 \\
51.200 \\
\end{tabular} & $\begin{array}{l}7.314 \\
7.314 \\
7.314 \\
7.314 \\
\end{array}$ & $\begin{array}{l}.027 \\
.027 \\
.027 \\
.027 \\
\end{array}$ \\
\hline $\begin{array}{l}\text { Error(Moment } \\
\text { Measurement) }\end{array}$ & $\begin{array}{l}\text { Sphericity Assumed } \\
\text { - Greenhouse-Geisser } \\
\text { Huynh-Feldt } \\
\text { Lower-bound }\end{array}$ & $\begin{array}{l}56.000 \\
56.000 \\
56.000 \\
56.000 \\
\end{array}$ & $\begin{array}{c}8 \\
8.000 \\
8.000 \\
8.000 \\
\end{array}$ & $\begin{array}{l}7.000 \\
7.000 \\
7.000 \\
7.000 \\
\end{array}$ & & \\
\hline
\end{tabular}

Tests of Between-Subjects Effects

Measure: Anxiety

Transformed Variable: Average

\begin{tabular}{|l|l|l|l|l|l|}
\hline Source & Sum of Squares & df & Mean Square & F & Sig. \\
\hline Intercept & 87384.200 & 1 & 87384.200 & 534.460 & .000 \\
Treatment & 57.800 & 1 & 57.800 & .354 & .000 \\
Error & 1308.000 & 8 & 163.500 & & \\
\hline
\end{tabular}

5. Are there statistically significant differences in anxiety in the different moments? $(\alpha=0.05)$ :

Ciclos de Mejora en el Aula (2020). Experiencias de Innovación Docente de la US Esta obra se distribuye con la licencia Creative Commons 

a) Yes
b) No
c) There is no enough information

6. Are there statistically significant differences in anxiety depending on the treatment? $(\alpha=0.05)$ (based on information presented in the tables)
a) Yes
b) No
c) There is not enough information

7. Are there statistically significant differences in anxiety in the different moments depending on the treatment? $(\alpha=0.05)$.
a) Yes
b) No
c) Thereis not enough information

8. How many different psychological treatments did we apply?
a) 4
b) 3
c) 2

9. Just in the stage of sphericity assumed, epsilon value is equal to:
a) 1
b) 8
c) 4

Ciclos de Mejora en el Aula (2020). Experiencias de Innovación Docente de la US Esta obra se distribuye con la licencia Creative Commons 


\title{
Cuestionario inicial-final para medir la evolución del estudiantado
}

\author{
A continuación, se presenta el cuestionario, tipo pre- \\ guntas abiertas referidas a dos casos prácticos, que se ha \\ aplicado al principio y al final del CIMA:
}

\section{Caso práctico 1:}

\begin{abstract}
We have a random sample of 21 individuals, randomly distributed into three groups of 7 participants each We want to test the effect of three forms of learning mathematical calculation (A, B, C) on performance.
\end{abstract} The results are the following:

\begin{tabular}{|l|l|l|}
\hline $\mathbf{A}$ & $\mathbf{B}$ & $\mathbf{C}$ \\
\hline 12 & 18 & 6 \\
\hline 18 & 17 & 4 \\
\hline 16 & 16 & 14 \\
\hline 8 & 18 & 4 \\
\hline 6 & 12 & 6 \\
\hline 12 & 17 & 12 \\
\hline 10 & 10 & 14 \\
\hline
\end{tabular}

Are there differences between different learning methods? Can you specify between which concrete groups? Answer this last question using Tukey and Scheffé. Which statistic is the most conservative? Why? $(\alpha=0.05)$

\section{Caso práctico 2:}

\begin{abstract}
We would like to study the effect of the variable Economic Level (econom), educative level (educat), kind of school (0:state; 1:private), nationality (nation; 0:Spanish; 1:non-Spanish) and gender ( $0:$ man; 1 woman), in math qualifications (in a sample of 260 children).

a) Determine the irrelevant variables (which do not correlate with the dependent variable), the redundant variables (which correlate with the dependent variable but share information with other independent variables, i.e. present multicollinearity).

b) Specify the variables that you would remove from the model and explain the reasons. Calculate the multiple $\mathrm{R}^{2}$ of the variables that present multicollinearity with the other independent variables.
\end{abstract}

We found the following results:

Ciclos de Mejora en el Aula (2020). Experiencias de Innovación Docente de la US Esta obra se distribuye con la licencia Creative Commons Reconocimiento-NoComercial-SinObraDerivada 4.0 Internacional (CC BY-NC-ND 4.0.) 
Coefficients ${ }^{\text {a }}$

\begin{tabular}{|c|c|c|c|c|c|c|c|c|c|c|}
\hline \multirow[b]{2}{*}{ Model } & & \multicolumn{2}{|c|}{$\begin{array}{l}\text { Non-standardized } \\
\text { coefficients }\end{array}$} & \multirow[b]{2}{*}{$\mathrm{t}$} & \multirow[b]{2}{*}{ Sig. } & \multicolumn{3}{|c|}{ Correlations } & \multicolumn{2}{|c|}{$\begin{array}{l}\text { Collinearity } \\
\text { statistics }\end{array}$} \\
\hline & & B & St. error & & & Zero order & Partial & Semipartial & Tolerance & VIF \\
\hline 1 & (Constant) & 51.654 & 3.369 & 15.334 & .000 & & & & & \\
\hline & econom & 1.968 & 1.332 & 1.478 & .141 & 621 & .092 & .068 & 157 & 6.356 \\
\hline & educat & 1.701 & .812 & 2.094 & .087 & .622 & .130 & .096 & .173 & 5.777 \\
\hline & school & -8.372 & 1.630 & -5.138 & .000 & -.611 & -.307 & -.236 & .515 & 1.941 \\
\hline & nation & .975 & 1.276 & .764 & .445 & .261 & .048 & .035 & .810 & 1.235 \\
\hline & gender & -.210 & 1.024 & -.205 & .838 & .017 & -.013 & -.009 & .998 & 1.002 \\
\hline
\end{tabular}

a. Dependent variable: math

Al final del CIMA, el alumnado también respondió a las siguientes preguntas subjetivas:

- "Valore de 1 (totalmente inútil) a 5 (totalmente útil) el uso de centro de calificaciones en las clases práctica para reforzar el aprendizaje".

- "Valore de 1 (nada motivante) a 5 (totalmente motivante) si la entrega de ejercicios y su corrección a través del centro de calificaciones le resultó motivante".

- “En qué contenido de las prácticas le hubiera gustado profundizar más?".

- "Por favor, explicite alguna mejora a realizar en las prácticas".

\section{Aplicación del CIMA}

\section{Relato resumido de las sesiones}

Las clases prácticas se imparten los lunes en dos subgrupos dentro de la asignatura de 9:30 a 11:30 y de 11:30 a 13:30. Normalmente estas clases suponen la resolución individual o en grupos de ejercicios prácticos tanto manualmente como con software estadístico. Por este motivo cuando las clases han sido presenciales se han impartido siempre en el aula informatizada. Una vez resueltos los ejercicios, el alumnado hace una puesta en común de cómo se ha resuelto, o sobre cómo resolverlo entre todos y todas las asistentes a clase.

Ciclos de Mejora en el Aula (2020). Experiencias de Innovación Docente de la US Esta obra se distribuye con la licencia Creative Commons 
La gran novedad de este curso ha sido la evaluación y seguimiento on-line de la resolución de ejercicios prácticos utilizando el centro de calificaciones de BlackBoard Collaborate Ultra. Normalmente esta entrega se realizaba otros años manualmente y por escrito. Esto ha supuesto que el alumnado ha dispuesto de una corrección inmediata de sus respuestas, lo que ha fomentado la puesta en común posterior en clases prácticas de las dudas y posibles discrepancias.

Para poder realizar esta aplicación del CIMA fue necesario la asistencia a cursos de formación on-line sobre aplicación de asignaturas al entorno virtual, módulos I y II, impartidos por la Universidad en Julio, con una evaluación en septiembre del 2020. Se usó la herramienta 'examen' de la opción evaluaciones BlackBoard Collaborate Ultra.

A pesar de que otros años también se daba esta entrega, pero in situ, este año ha supuesto una mayor participación y respuesta de todo el alumnado que asiduamente asiste a prácticas. Téngase presente que había un calendario con fecha y hora de entrega programadas en la aplicación.

Ha sido interesante constatar que el 100\% del alumnado que ha seguido en la asignatura (que no se ha cambiado de grupo o ha abandonado) ha participado en todas las entregas $(n=20)$. A pesar de que un $20 \%$ de ellos no asistió a todas las sesiones prácticas

\section{Evaluación del aprendizaje del alumnado durante el CIMA}

El pretest ha sido cumplimentado por el alumnado el primer día de clase de la asignatura ( 6 de octubre). Del análisis de respuestas del cuestionario inicial (de respuesta

Ciclos de Mejora en el Aula (2020). Experiencias de Innovación Docente de la US Esta obra se distribuye con la licencia Creative Commons 
abierta siguiendo las sugerencias de Rivero y Porlán, 2017), se ha obtenido una escalera de conocimiento. Nuestra hipótesis era que, al final del CIMA, los conocimientos habrían aumentado.

Para fomentar la participación en el pretest y post-test, esta se ha valorado con 0,5 en la calificación de la asignatura. El post-test se ha realizado el 24 de noviembre.

Ambas pruebas se han aplicado a través de Enseñanza Virtual, las respuestas las hemos registrado a través del centro de calificaciones, en el formato encuesta. Debido a cómo funciona la aplicación, las respuestas del alumnado han sido anónimas.

A continuación, en la tabla 1, se presenta la escalera de aprendizaje del pretest y la evolución que se encontró en el post-test. Las diferentes respuestas se presentan en orden, desde la más completa a la más incorrecta.

Tabla 1. Escalera de aprendizaje. Comparativa entre pretest y post-test

\begin{tabular}{|c|c|c|c|}
\hline Preguntas abiertas & Respuestas & $\%$ pre & $\%$ post \\
\hline \multirow{6}{*}{$\begin{array}{l}\text { Are there differences } \\
\text { between different } \\
\text { learning methods? } \\
\text { Can you specify } \\
\text { between which } \\
\text { concrete groups? } \\
\text { Answer this last } \\
\text { question using Tukey } \\
\text { and Scheffé. Which } \\
\text { statistic is the most } \\
\text { conservative? Why? } \\
(\alpha=0.05)\end{array}$} & $\begin{array}{l}\text { Respuestas correctas con } \\
\text { argumentación adecuada }\end{array}$ & - & 75 \\
\hline & $\begin{array}{l}\text { Respuestas correctas con } \\
\text { argumentación parcial }\end{array}$ & - & 25 \\
\hline & $\begin{array}{l}\text { Respuestas aproximadas con } \\
\text { argumentación parcial }\end{array}$ & 25 & - \\
\hline & Respuesta incorrecta sin justificar & 25 & - \\
\hline & Sin respuesta alguna & 50 & - \\
\hline & & & \\
\hline
\end{tabular}

Ciclos de Mejora en el Aula (2020). Experiencias de Innovación Docente de la US Esta obra se distribuye con la licencia Creative Commons 
Determine:

- the irrelevant variables.

- the redundant variables.

- Specify the variables that you would remove.

- multicollinearity with the other independent variables.

n=20 (alumnado que realizó prueba previa y posterior).

Puede apreciarse como claramente ha habido una evolución muy positiva por parte del alumnado desde la prueba previa a la posterior. No obstante, se observa que respecto al segundo caso práctico los conceptos no han sido tan bien adquiridos como respecto al primero de ellos. Es de entender, ya que se trata de conceptos más complejos y menos trabajados en clase al formar parte de la parte final del contenido impartido en segunda quincena de noviembre. Este dato en todo caso es de interés para seguir y organizar la marcha del curso hasta finales del cuatrimestre a finales de enero de 2021.

\section{Evaluación de mi diseño e intervención}

Mi diseño e intervención del CIMA se ha efectuado del siguiente modo:

(a) Diario de clase: en las exposiciones del alumnado he ido registrando posibles incidencias, prestando especial atención a las problemáticas comunes que se han ido presentando en las distintas clases y a las casuísticas relacionadas con cada tipo de ejercicio práctico desarrollado. Esta práctica me ha servido para poner dichas incidencias en común con el alumnado para analizar si realmente se

Ciclos de Mejora en el Aula (2020). Experiencias de Innovación Docente de la US Esta obra se distribuye con la licencia Creative Commons 
trataba de algún problema a resolver o había sido un 'falso positivo'. Algo que des de esta dinámica surgió fue la comunicación de incidencias por parte del alumnado de las que yo no era consciente. Por ejemplo, las dificultades para poder descargarse el software estadístico desde la página de la universidad, o los errores en las grabaciones de las sesiones prácticas disponibles en la plataforma. Esto facilitó la resolución de incidencias de una manera rápida y eficaz lo que posibilitó una mejor marcha de las prácticas y recursos disponibles para el alumnado.

(b) Evaluación 'in situ' de cada entrega realizada en el centro de calificaciones. Se recogerá el porcentaje de respuestas correctas. Esta actuación ha tenido muchísima mayor incidencia de la esperada. Principalmente por dos motivos, primero porque ha aumentado la participación del alumnado. Al ser la entrega on-line, esto ha flexibilizado la entrega y por tanto la participación, ya que la entrega no necesariamente se tenía que realizar en el momento de las clases, siendo el tiempo de entrega hasta las 16:00 del día de la práctica. En segundo lugar, el disponer de una corrección y valoración automática de puntos cuantificables para la calificación final, aumentaba el interés por la puesta en común de la resolución de casos en clases prácticas.

(c) Opinión del alumnado sobre una serie de preguntas realizadas al final del post-test. En este caso la respuesta no ha sido tan alta, han participado en la encuesta 15 personas (75\% del alumnado que ha participado activamente en la actividad). Los resultados has sido satisfactorios.

Respecto a la pregunta "Valore de 1 (totalmente inútil) a 5 (totalmente útil) el uso de centro de calificaciones en las clases práctica para reforzar el aprendizaje". Se ha obtenido una mediana de 4.

Ciclos de Mejora en el Aula (2020). Experiencias de Innovación Docente de la US Esta obra se distribuye con la licencia Creative Commons 
Respecto a la pregunta "Valore de 1 (nada motivante) a 5 (totalmente motivante) si la entrega de ejercicios y su corrección a través del centro de calificaciones le resultó motivante", se ha obtenido una mediana de 3,5.

Respecto a la pregunta “¿En qué contenido de las prácticas le hubiera gustado profundizar más?", En este caso, las sugerencias han estado focalizadas en que se aumenten las prácticas sobre 'contrastes ortogonales a priori' en el análisis de la varianza.

Y, por último, respecto a la pregunta "Por favor, explicite alguna mejora a realizar en las prácticas". En este caso, las sugerencias se han focalizado en dos cuestiones, una en aumentar los tiempos de descanso, y una segunda en hacer más número de ejercicios de exámenes.

\section{Cuestiones a mantener y cambios a introducir}

En síntesis, después de esta experiencia de CIMA, seguiremos manteniendo la dinámica de evaluación y seguimiento on-line de la resolución de ejercicios prácticos utilizando el centro de calificaciones de BlackBoard Collaborate Ultra, ya que el alumnado lo ha encontrado útil, no obstante, consideramos que hemos de mejorar la motivación incluyendo casos prácticos, como videos de noticias actuales, o estadísticas controvertidas que hagan más atrayentes las dinámicas de clase.

Respecto a cuestiones metodológicas-procedimentales, debemos mejorar la identificación del alumnado y los sistemas de registro, ya que debido a problemas en el sistema y posiblemente debido a nuestra inexperiencia, un porcentaje importante de alumnado entrego el post-test por correo electrónico. Lo que ha implicado la recodificación de sus repuestas en la base de datos Excel manualmente.

Ciclos de Mejora en el Aula (2020). Experiencias de Innovación Docente de la US Esta obra se distribuye con la licencia Creative Commons 


\section{Aspectos a incorporar en la práctica habitual}

Un aspecto que me gustaría destacar para la práctica docente habitual es la necesidad de desarrollar 'feedback' explícito, empírico, operacionalizado y continuo del alumnado. Esto facilita el diseño e implementación adecuada de la materia en consonancia con el progreso real del alumnado.

\section{Principios didácticos que han guiado esta experiencia y que deben permanecer en el futuro}

En último término resaltar que los principios didácticos argumentados que han guiado la experiencia presente y que deben permanecer en el futuro han de promover la participación activa (García-Pérez \& Porlán, 2017) y la motivación del alumnado. Pero este foco debe ser operativo con el registro de datos que luego se comparta y valore con el alumnado durante el proceso.

Ciclos de Mejora en el Aula (2020). Experiencias de Innovación Docente de la US Esta obra se distribuye con la licencia Creative Commons 
Palabras clave: “Diseño y Análisis de Datos en Psicología II", "Grado en Psicologia”, "Docencia universitaria", "Experimentación docente universitaria", "Evaluación continua universitaria on-line".

Keywords: "Design and Analysis of Data in Psychology II", "Degree in Psychology", "University teaching", "University teaching experimentation", "On-line continuous evaluation in university".

\section{Referencias bibliográficas}

Chacón, S. (2018). Trabajo en grupo en base a ejercicios de formato cerrado. En V Jornadas de Docencia Universitaria (pp. 146-160). Sevilla: Instituto de Ciencias de la Educación de la Universidad de Sevilla. Disponible en http://dx.doi.org/10.12795/JDU.2018.i01.08

Chacón, S. (2020). Participación activa del alumnado en el proceso de enseñanza en Psicometría. En E. Navarro-Medina y R. Porlán Ariza (Eds.), Ciclos de Mejora en el aula. Año 2019. Experiencias de innovación docente de la Universidad de Sevilla (pp. 1839-1850). Sevilla, España: Editorial Universidad de Sevilla. Disponible en http://dx.doi.org/10.12795/9788447221912.080

García-Díaz, E., Porlán, R. y Navarro, E. (2017). Los fines y los contenidos de enseñanza. En R. Porlán (Coord.), Enseñanza Universitaria. Cómo mejorarla (pp. 93-104). Madrid, España: Morata.

García-Pérez, F. y Porlán, R. (2017). Los principios didácticos y el modelo didáctico personal. En R. Porlán (Coord.), Enseñanza Universitaria. Cómo mejorarla (pp. 93-104). Madrid, España: Morata.

Polanco-Hernández, A. (2005). La motivación en los estudiantes universitarios. Actualidades Investigativas en Educación, 5(2), 1-13.

Rivero, A. y Porlán, R. (2017). La evaluación en la enseñanza universitaria. En R. Porlán (Coord.), Enseñanza Universitaria. Cómo mejorarla (pp. 73-91). Madrid, España: Morata.

Ciclos de Mejora en el Aula (2020). Experiencias de Innovación Docente de la US Esta obra se distribuye con la licencia Creative Commons 